\title{
ALGEBRAS WHOSE RIGHT NUCLEUS IS A CENTRAL SIMPLE ALGEBRA
}

\author{
S. PUMPLÜN
}

\begin{abstract}
We generalize Amitsur's construction of central simple algebras over a field $F$ which are split by field extensions possessing a derivation with field of constants $F$ to nonassociative algebras: for every central division algebra $D$ over a field $F$ of characteristic zero there exists an infinite-dimensional unital nonassociative algebra whose right nucleus is $D$ and whose left and middle nucleus are a field extension $K$ of $F$ splitting $D$, where $F$ is algebraically closed in $K$.

We then give a short direct proof that every $p$-algebra of degree $m$, which has a purely inseparable splitting field $K$ of degree $m$ and exponent one, is a differential extension of $K$ and cyclic. We obtain finite-dimensional division algebras over a field $F$ of characteristic $p>0$ whose right nucleus is a division $p$-algebra.
\end{abstract}

\section{INTRODUCTION}

In 1954, Amitsur [2] observed that all associative central division algebras over a field $F$ of characteristic zero can be constructed using differential polynomials. His construction method can be considered as an analogue to the the well known crossed product construction, except that he uses splitting fields $K$ of the algebras, where the base field $F$ is algebraically closed in $K$, instead of their algebraic splitting fields. Some of his results also work for $p$-algebras, i.e. over base fields of characteristic $p>0$.

In this paper, we consider algebras which are also obtained from differential polynomials, but which are nonassociative.

These algebras are constructed using the differential polynomial ring $K[t ; \delta]$, where $K$ is a field and $\delta$ a derivation on $K$ and were defined by Petit [14]: given a differential polynomial $f \in K[t ; \delta]$ of degree $m$, the set of all differential polynomials of degree less than $m$, together with the addition given by the usual addition of polynomials, can be equipped with a nonassociative ring structure using right division by $f$ to define the multiplication as $g \circ h=$ $g h \bmod _{r} f$. The resulting nonassociative unital ring $S_{f}$, also denoted by $K[t ; \delta] / K[t ; \delta] f$, is an algebra over the field of constants $F=\operatorname{Const}(\delta)$ of $\delta$. If $f$ generates a two-sided ideal in $K[t ; \delta]$, then $S_{f}$ is the (associative) quotient algebra obtained by factoring out the two-sided principal ideal generated by $f$.

If $f$ is not two-sided and $\delta$ not trivial, then the nuclei of $S_{f}$ are larger than the center $F=\operatorname{Const}(\delta)$. In that case the left and middle nucleus are always given by $K$, whereas the right nucleus reflects both the choice of $f$ and the structure of the ring $K[t ; \delta]$.

1991 Mathematics Subject Classification. Primary: 17A35; Secondary: 17A60, 16 S36.

Key words and phrases. Differential polynomial ring, skew polynomial, differential polynomial, differential operator, differential algebra, nonassociative algebra, right nucleus. 
We proceed as follows: The basic terminology and notation we use can be found in [2] and Section 1. Section 2 rephrases some of Amitsur's results for those algebras $S_{f}$ which have a central simple algebra as their right nucleus. For this we employ Amitsur's $A$-polynomials. In Sections 3 and 4 we show how to construct algebras $S_{f}$ with a given central simple algebra as right nucleus, first for base fields of characteristic zero, then for base fields of characteristic $p>0$ : for every central simple algebra $B$ of degree $m$ over a field $F$ of characteristic zero which is split by a field extension $K / F$ in which $F$ is algebraically closed, there exists an infinite-dimensional unital algebra $S_{f}=K[t ; \delta] / K[t ; \delta] f$ over $F$ with right nucleus $B$ (and left and middle nucleus $K$ ), see Theorem 8. In particular, for every central division algebra $D$ over $F$ there exists an infinite-dimensional unital algebra $S_{f}$ over $F$ with right nucleus $D$ (Corollary 9).

We present a short proof that every $p$-algebra $B$ of degree $m$ over a field $F$ of characteristic $p$ which is split by a purely inseparable field extension $K / F$ of exponent one and degree $m$ is isomorphic to a differential extension $\left(K, \delta, d_{0}\right)$ of $K$ (Theorem 13), only invoking a result on the structure of $S_{f}$ and Amitsur's [2, Lemma 20']. Thus it is cyclic by [9, Main Theorem].

For every division $p$-algebra $D$ of degree $m$ over a field $F$ of characteristic $p$ which is split by a purely inseparable field extension $K / F$ of exponent one such that $m<[K: F]$, there is a unital division algebra $S_{f}=K[t ; \delta] / K[t ; \delta] f$ over $F$ of dimension $m p^{e}$ with right nucleus $D$ and left and middle nucleus $K$. The smallest possible dimension $l$ of such a division algebra containing $D$ as right nucleus is bounded via $m^{2}<l \leq m p^{m-1}$ and connected to the number of cyclic algebras that are needed when expressing $D$ as a product of cyclic algebras of degree $p$ in the Brauer group $\operatorname{Br}(F)$ (Corollary 18).

\section{Preliminaries}

1.1. Nonassociative algebras. Let $F$ be a field and let $A$ be an $F$-vector space. $A$ is an algebra over $F$ if there exists an $F$-bilinear map $A \times A \rightarrow A,(x, y) \mapsto x \cdot y$, denoted simply by juxtaposition $x y$, the multiplication of $A$. An algebra $A$ is called unital if there is an element in $A$, denoted by 1 , such that $1 x=x 1=x$ for all $x \in A$. We will only consider unital algebras from now on without explicitly saying so.

An algebra $A \neq 0$ is called a division algebra if for any $a \in A, a \neq 0$, the left multiplication with $a, L_{a}(x)=a x$, and the right multiplication with $a, R_{a}(x)=x a$, are bijective. If $A$ has finite dimension over $F, A$ is a division algebra if and only if $A$ has no zero divisors [17, pp. $15,16]$.

Associativity in $A$ is measured by the associator $[x, y, z]=(x y) z-x(y z)$. The left nucleus of $A$ is defined as $\operatorname{Nuc}_{l}(A)=\{x \in A \mid[x, A, A]=0\}$, the middle nucleus of $A$ is $\operatorname{Nuc}_{m}(A)=$ $\{x \in A \mid[A, x, A]=0\}$ and the right nucleus of $A$ as $\operatorname{Nuc}_{r}(A)=\{x \in A \mid[A, A, x]=$ $0\}$. $\operatorname{Nuc}_{l}(A), \operatorname{Nuc}_{m}(A)$, and $\operatorname{Nuc}_{r}(A)$ are associative subalgebras of $A$. Their intersection $\operatorname{Nuc}(A)=\{x \in A \mid[x, A, A]=[A, x, A]=[A, A, x]=0\}$ is the nucleus of $A$. Nuc $(A)$ is an associative subalgebra of $A$ containing $F 1$ and $x(y z)=(x y) z$ whenever one of the elements $x, y, z$ is in $\operatorname{Nuc}(A)$. The center of $A$ is $\mathrm{C}(A)=\{x \in \operatorname{Nuc}(A) \mid x y=y x$ for all $y \in A\}$. 
1.2. Differential polynomial rings. Let $K$ be a field and $\delta: K \rightarrow K$ a derivation, i.e. an additive map such that

$$
\delta(a b)=a \delta(b)+\delta(a) b
$$

for all $a, b \in K$. The differential polynomial ring $K[t ; \delta]$ is the set of polynomials

$$
a_{0}+a_{1} t+\cdots+a_{n} t^{n}
$$

with $a_{i} \in K$, where addition is defined term-wise and multiplication by

$$
t a=a t+\delta(a) \quad(a \in K) .
$$

For $f=a_{0}+a_{1} t+\cdots+a_{n} t^{n}$ with $a_{n} \neq 0$ define $\operatorname{deg}(f)=n$ and $\operatorname{deg}(0)=-\infty$. Then $\operatorname{deg}(f g)=\operatorname{deg}(f)+\operatorname{deg}(g)$. An element $f \in R$ is irreducible in $R$ if it is not a unit and if it has no proper factors, i.e if there do not exist $g, h \in R$ with $\operatorname{deg}(g), \operatorname{deg}(h)<\operatorname{deg}(f)$ such that $f=g h$.

$R=K[t ; \delta]$ is a left and right principal ideal domain and there is a right division algorithm in $R$ : for all $g, f \in R, g \neq 0$, there exist unique $r, q \in R$ with $\operatorname{deg}(r)<\operatorname{deg}(f)$, such that $g=q f+r$. There is also a left division algorithm in $R$ [11, p. 3 and Prop. 1.1.14]. (Our terminology is the one used by Petit [14]; Jacobson's is vice versa.)

Two non-zero elements $f, g \in R$ are called similar $(f \sim g)$ if and only if there exist $h, q, u \in R$ such that

$$
1=h f+q g \text { and } u^{\prime} f=g u
$$

for some $u^{\prime} \in R$. Equivalently, $f$ and $g$ are similar if $R / R f$ and $R / R g$ are isomorphic as $R$-modules [11, p. 11]. Obviously, $f \sim g$ implies that $\operatorname{deg}(f)=\operatorname{deg}(g)$.

1.3. The characteristic $p>0$ case. Let $K$ be a field of characteristic $p$ and $R=K[t ; \delta]$, then

$$
(t-b)^{p}=t^{p}-V_{p}(b), \quad V_{p}(b)=b^{p}+\delta^{p-1}(b), \quad(t-b)^{p^{e}}=t^{p^{e}}-V_{p^{e}}(b)
$$

for all $b \in K$ with $V_{p^{e}}(b)=V_{p}^{e}(b)=V_{p}\left(\ldots\left(V_{p}(b)\right) \ldots\right)[11$, p. 17ff]. For any $p$-polynomial

$$
f(t)=a_{0} t^{p^{e}}+a_{1} t^{p^{e-1}}+\cdots+a_{e} t+d \in D[t ; \delta]
$$

we thus have

$$
f(t)-f(t-b)=a_{0} V_{p^{e}}(b)+a_{1} V_{p^{e-1}}(b)+\cdots+a_{e} b
$$

for all $b \in K$ and define

$$
V_{f}(b)=a_{0} V_{p^{e}}(b)+a_{1} V_{p^{e-1}}(b)+\cdots+a_{e} b .
$$

1.4. Nonassociative algebras obtained from differential polynomial rings. Let $K$ be a field and $f \in R=K[t ; \delta]$ of degree $m$. Let $\bmod _{r} f$ denote the remainder of right division by $f$. Define $F=\operatorname{Cent}(\delta)=\{a \in K \mid \delta(a)=0\}$.

Definition 1. (cf. $[14,(7)])$ The vector space

$$
R_{m}=\{g \in K[t ; \delta] \mid \operatorname{deg}(g)<m\}
$$

together with the multiplication

$$
g \circ h=g h \bmod _{r} f
$$


is a unital nonassociative algebra $S_{f}=\left(R_{m}, \circ\right)$ over

$$
F_{0}=\left\{a \in K \mid a h=h a \text { for all } h \in S_{f}\right\} .
$$

$F_{0}$ is a subfield of $K[14,(7)]$ and it is easy to check that $F_{0}=\operatorname{Cent}(\delta)$. The algebra $S_{f}$ is also denoted by $R / R f[14,16]$ if we want to make clear which ring $R$ is involved in the construction. In the following, we call the algebras $S_{f}$ Petit algebras and denote their multiplication simply by juxtaposition. Without loss of generality, we may assume that $f$ is monic, since $S_{f}=S_{g}$ for all $g=a f$ with $a \in K^{\times}$.

Using left division by $f$ and the remainder $\bmod _{l} f$ of left division by $f$ instead, we can define the multiplication for another unital nonassociative algebra on $R_{m}$ over $F$, called ${ }_{f} S$ or $R / f R$. We will only consider the Petit algebras $S_{f}$, however, since every algebra ${ }_{f} S$ is the opposite algebra of some Petit algebra (cf. [14, (1)]).

Right multiplication with $0 \neq g \in S_{f}$ is given by $R_{g}: S_{f} \longrightarrow S_{f}, h \mapsto h g$, and is a left $K$-module endomorphism. Left multiplication $L_{g}: S_{f} \longrightarrow S_{f}, h \mapsto g h$ is an $F$-module endomorphism [14], and if we view $S_{f}$ as a right module over $\operatorname{Nuc}_{r}\left(S_{f}\right)$, a right $\operatorname{Nuc}_{r}\left(S_{f}\right)$ module endomorphism.

Clearly $S_{f}$ has no zero divisors if and only if $R_{g}$ and $L_{g}$ are injective.

Theorem 1. (cf. [14, (2), p. 13-03, (5), (6), (7), (9), (14)]) Let $f \in R=K[t ; \delta]$.

(i) If $S_{f}$ is not associative then $\operatorname{Nuc}_{l}\left(S_{f}\right)=\operatorname{Nuc}_{m}\left(S_{f}\right)=K$ and

$$
\operatorname{Nuc}_{r}\left(S_{f}\right)=\left\{g \in R_{m} \mid f g \in R f\right\} .
$$

The right nucleus of $S_{f}$ is Amitsur's invariant ring of $f$.

(ii) The powers of $t$ are associative if and only if $t^{m} t=t t^{m}$ if and only if $t \in \operatorname{Nuc}_{r}\left(S_{f}\right)$ if and only if $f t \in R f$.

(iii) If $f$ is irreducible then $\operatorname{Nuc}_{r}\left(S_{f}\right)$ is an associative division algebra.

(iv) Let $f \in R$ be irreducible and $S_{f}$ a finite-dimensional $F$-vector space or free of finite rank as a right $\operatorname{Nuc}_{r}\left(S_{f}\right)$-module. Then $S_{f}$ is a division algebra.

Conversely, if $S_{f}$ is a division algebra then $f$ is irreducible.

(v) $S_{f}$ is associative if and only if $f$ is a two-sided element (i.e., generates a two-sided ideal $R f)$. In that case, $S_{f}$ is the usual quotient algebra $K[t ; \delta] /(f)$.

(vi) $f$ is irreducible if and only if $S_{f}$ is a right division algebra over $F$ (i.e., each non-zero element in $S_{f}$ has a left inverse: there is $z \in S_{f}$ such that $z h=1$ ), if and only if $S_{f}$ has no zero divisors.

Recall that a polynomial $f \in R=K[t ; \delta]$ is bounded if there exists $0 \neq f^{*} \in R$, such that $R f^{*}=f^{*} R$ is the largest two-sided ideal of $R$ contained in $R f$.

If $f \in R$ is bounded then $f$ is irreducible if and only if $\operatorname{Nuc}_{r}\left(S_{f}\right)$ has no zero divisors if and only if $\operatorname{Nuc}_{r}\left(S_{f}\right)$ is an associative division algebra (cf. [8, Proposition 4] which sums up classical results from [10]). [5, Theorem 4] yields:

Theorem 2. Let $f \in R$ be irreducible. Then $f$ is bounded if and only if $S_{f}$ is free of finite rank as a $\mathrm{Nuc}_{r}\left(S_{f}\right)$-module. In this case, $S_{f}$ is a division algebra. 
Proof. The first part of the statement is [5, Theorem 4]. Since $f$ irreducible, $S_{f}$ is a right division algebra and $L_{h}$ is injective for all $h \in S_{f}, h \neq 0$, as observed in [14, Section 2., (7)]. The second part then follows from the fact that $S_{f}$ is free of finite rank as a $\operatorname{Nuc}_{r}\left(S_{f}\right)$ module, which means the injective $\operatorname{Nuc}_{r}\left(S_{f}\right)$-linear map $L_{h}$ is also surjective.

$R=K[t ; \delta]$ has finite rank over its center if and only if $K$ is of finite rank over $C_{t}=$ $\{a \in K \mid a t=t a\}$ if and only if all polynomials of $R$ are bounded and if for all $f$ of degree non-zero, $\operatorname{deg}\left(f^{*}\right) / \operatorname{deg}(f)$ is bounded in $\mathbb{Q}\left(f^{*}\right.$ being the bound of $\left.f\right)[6$, Theorem IV]. Since here $C_{t}=\operatorname{Const}(\delta)=F$, we conclude:

Proposition 3. Assume that one of the two following equivalent conditions hold:

(i) $R=K[t ; \delta]$ has finite rank over its center;

(ii) $K / F$ is a finite field extension.

Then every $f \in R$ is bounded. In particular, if $f$ is irreducible then $S_{f}$ is a division algebra.

Note that if $K / F$ is a finite field extension then the derivation $\delta$ is trivial, or $K$ has characteristic $p>0$.

We will assume throughout the paper that $f \in K[t ; \delta] \operatorname{has} \operatorname{deg}(f)=m \geq 2$ (if $f$ has degree $m=1$ then $S_{f} \cong K$ ) and that $\delta \neq 0$. Without loss of generality, we could only look at monic $f$, but will do so only when explicitly mentioned.

\section{NonAssociative Algebras Whose Right NUCleus is A CENTRAL Simple Algebra}

We use the terminology from [2] with the only exception that that in our definition of $K[t ; \delta]$, we look at polynomials with the coefficients written on the left, not on the righthand-side as in [2]. All results, however, work analogously in this case.

By [13, Theorem 4.2], given a field extension $K / F$ in characteristic zero, $F$ is the field of constants of a derivation of $K$ if and only if $F$ is algebraically closed in $K$.

In this section, let $K$ be a field of characteristic 0 . Let $\delta$ be a derivation of $K$ with $F=\operatorname{Const}(\delta)$ and $f \in R=K[t ; \delta]$. The finite-dimensional associative $F$-algebra $\operatorname{Nuc}_{r}\left(S_{f}\right)$ is called the invariant ring of $f$ by Amitsur [2, p. 260], in recent literature it is also referred to as the eigenspace of $f$.

Let $V$ be an $K$-vector space. An additive map $T: V \longrightarrow V$, such that $T(\alpha v)=\alpha T(v)+$ $\delta(\alpha) v$ for all $v \in V$ and $\alpha \in K$, is called a pseudo-linear transformation on $V$. Given a basis of $V$, a pseudo-linear transformation $T$ on $V$ is given by a matrix. Moreover, $(V, T)$ is isomorphic to $K[t ; \delta] / f(t) K[t ; \delta]$ for some $f(t) \in K[t ; \delta]$ which is called the characteristic polynomial of $T$ [2, p. 250]. The characteristic polynomial is uniquely determined up to similarity and any polynomial $f(t)$ is the characteristic polynomial of some pseudo-linear transformation $(V, T)$ (simply define $V=K[t ; \delta] / K[t ; \delta] f(t)$ and $T(p(t)+K[t ; \delta] f(t))=$ $t p(t)+K[t ; \delta] f(t))$.

Let $(V, T)$ and $\left(V^{\prime}, T^{\prime}\right)$ be two pseudo-linear transformations with characteristic polynomials $f, g \in K[t ; \delta]$ where $\operatorname{deg}(f)=m$ and $\operatorname{deg}(g)=n$. Then there is a pseudo-linear transformation $T \times T^{\prime}$ on the tensor product $V \otimes V^{\prime}$ defined via

$$
\left(T \times T^{\prime}\right)(u)=\sum_{i} T\left(v_{i}\right) \otimes w_{i}+\sum_{i} v_{i} \otimes T^{\prime}\left(w_{i}\right)
$$


for all $u=\sum_{i} v_{i} \otimes w_{i} \in V \otimes V^{\prime}$.

Furthermore, let $f, g \in K[t ; \delta]$ where $\operatorname{deg}(f)=m$ and $\operatorname{deg}(g)=n$, and $T$ and $T^{\prime}$ be the pseudo-linear transformation defined using $f$ and $g$. Then the resultant $f \times g$ of $f$ and $g$ is any characteristic polynomial of $T \times T^{\prime}$, so that $f \times g$ is a polynomial of degree $n m$ uniquely determined up to similarity [2, p. 255].

A differential polynomial $f \in K[t ; \delta]$ of degree $m$ is called an $A$-polynomial if there is some $\tilde{f} \in K[t ; \delta]$ of degree $n$ such that the resultant $f \times \tilde{f}$ is similar to $e_{m n}$, the characteristic polynomial of the pseudo-linear transformation corresponding to the zero $m n \times m n$ matrix [2, p. 263].

Amitsur's results tell us when $\operatorname{Nuc}_{r}\left(S_{f}\right)$ is a central simple algebra:

Theorem 4. [2, Lemma 17, 18, 19, Theorem 17, Corollary, Lemma 22] Let $f, g \in K[t ; \delta]$ with $\operatorname{deg}(f)=m \geq 2$ and $\operatorname{deg}(g)=n \geq 2$.

(i) $\operatorname{Nuc}_{r}\left(S_{f}\right)$ has dimension $m^{2}$ if and only if $f$ is an A-polynomial.

(ii) If $f$ is an A-polynomial then $\operatorname{Nuc}_{r}\left(S_{f}\right)$ is a central simple algebra of degree $m$ which is split by $K$.

(iii) If $f$ and $g$ are A-polynomials then so is $h=f \times g$ and

$$
\operatorname{Nuc}_{r}\left(S_{h}\right)=\operatorname{Nuc}_{r}\left(S_{f}\right) \otimes_{F} \operatorname{Nuc}_{r}\left(S_{g}\right) .
$$

(iv) If $f$ and $g$ are A-polynomials then

$$
\operatorname{Nuc}_{r}\left(S_{f}\right) \cong \operatorname{Nuc}_{r}\left(S_{g}\right)
$$

if and only if $f \sim g(t+a) \sim g(t) \times t+a$ for some $a \in K$. In particular,

$$
S_{f} \cong S_{g} \text { implies that } f \sim g(t+a) \sim g(t) \times t+a
$$

for some $a \in K$.

(v) Suppose $f$ is an A-polynomial. Then

$$
\operatorname{Nuc}_{r}\left(S_{f}\right) \cong \operatorname{Mat}_{m}(F)
$$

if and only if one of the following holds:

- $f \sim e_{m} \times t+c$ for some $c \in K$;

- $f$ decomposes into irreducible factors and at least one factor is linear of the form $t+c$ for some $c \in K$ (then $f \sim e_{m} \times t+c$ ).

In particular, then the irreducible factors of $f$ are all similar to $t+c$.

Let $L / K$ be a field extension such that $\delta$ extends to $L$. Then $L[t ; \delta]$ is an Ore extension of $K[t ; \delta]$ and the constant field $F=\operatorname{Const}\left(\left.\delta\right|_{K}\right)$ of $\delta=\left.\delta\right|_{K}$ is contained in the constant field $C=\operatorname{Const}(\delta)$. If $L=K \cdot C$ is the composite field of $K$ and $C$, we say $L$ is a constant extension of $K$. It is clear that for $f \in K[t ; \delta]$,

$$
\operatorname{Nuc}_{r}(K[t ; \delta] / K[t ; \delta] f) \subset \operatorname{Nuc}_{r}(L[t ; \delta] / L[t ; \delta] f) .
$$

Theorem 5. Let $f \in K[t ; \delta]$ be of degree $m$ and $L / K$ a field extension such that $\delta$ extends to $L$ and $C=\operatorname{Const}(\delta)$. Suppose that $L$ is a constant extension of $K$.

(i) If $f$ is an A-polynomial then $f \in L[t ; \delta]$ is an A-polynomial and

$$
\operatorname{Nuc}_{r}(L[t ; \delta] / L[t ; \delta] f) \cong \operatorname{Nuc}_{r}(K[t ; \delta] / K[t ; \delta] f) \otimes_{F} C .
$$


(ii) Suppose $B=\operatorname{Nuc}_{r}\left(S_{f}\right)$ is a central simple algebra of degree $m$ over $F$ with $f \in K[t ; \delta]$. Then $C$ splits $B$ if and only if $f$ has a left or right root in $L$, i.e. $f=(t-a) g(t) \in L[t ; \delta]$ or $f=g(t)(t-a) \in L[t ; \delta]$.

In particular, then $S_{f} \otimes_{F} C$ has right nucleus $\operatorname{Mat}_{m}(C)$.

This follows from [2, Theorem 20] and [2, Corollary, p. 270].

Remark 6. Since every automorphism of a nonassociative algebra maps the right nucleus onto itself, for every $A$-polynomial $f$ which is not two-sided, each $H \in \operatorname{Aut}_{F}\left(S_{f}\right)$ satisfies $\left.H\right|_{B} \in \operatorname{Aut}_{F}(B)$ when restricted to the central simple algebra $B=\operatorname{Nuc}_{r}\left(S_{f}\right)$, thus $\left.H\right|_{B}$ is an inner automorphism of $B$. By an analogous argument, also $\left.H\right|_{K} \in \operatorname{Aut}_{F}(K)$.

\section{Algebras Whose Right NUCleus is SPlit By AN EXTENSION IN Which $F$ is} ALGEBRAICALLY CLOSED

Let $F$ be a field of characteristic 0 .

Theorem 7. [2, Lemma 20] (i) Every central simple algebra $B$ of degree $m$ over $F$ which is split by a field extension $K / F$ in which $F$ is algebraically closed, is isomorphic to $\operatorname{Nuc}_{r}\left(S_{f}\right)$ for some $f \in K[t ; \delta]$ of degree $m$ and a suitable $\delta$ with $F=\operatorname{Const}(\delta)$. The differential polynomial $f$ is an A-polynomial.

(ii) Every central division algebra $D$ of degree $m$ over $F$ is isomorphic to $\operatorname{Nuc}_{r}\left(S_{f}\right)$ for some $f \in K[t ; \delta]$ of degree $m$ and a suitable differential field $(K, \delta)$.

Note that (ii) follows from (i), since for every central division algebra $D$ over $F$, the function field $K(X)$ of the Severi-Brauer variety $X$ of $D$ splits $D$ ([2, p. 245] or [3]), and we can always find a derivation $\delta$ on $K(X)$ with $F=\operatorname{Const}(\delta)$, as $F$ is algebraically closed in $K(X)$.

As an immediate consequence of Theorem 7 and Remark 6, we now get the following results:

Theorem 8. For every central simple algebra $B$ of degree $m$ over $F$ which is split by a field extension $K / F$ in which $F$ is algebraically closed, there is a derivation $\delta$ on $K$ with field of constants $F$ and a differential polynomial $f \in K[t ; \delta]$ of degree $m$, such that

$$
S_{f}=K[t ; \delta] / K[t ; \delta] f
$$

is an infinite-dimensional algebra over $F$ with right nucleus $B$ and left and middle nucleus $K$. Every automorphism $H \in \operatorname{Aut}_{F}\left(S_{f}\right)$ extends an inner automorphism of $B$.

We conclude from [2, p. 246]:

Corollary 9. For every central division algebra $D$ of degree $m$ over $F$, there exists a field extension $K / F$ in which $F$ is algebraically closed, a derivation $\delta$ on $K$ with field of constants $F$, and a differential polynomial $f \in K[t ; \delta]$ of degree $m$, such that

$$
S_{f}=K[t ; \delta] / K[t ; \delta] f
$$

is an infinite-dimensional algebra over $F$ with right nucleus $D$, and left and middle nucleus $K$. $K$ splits $D$ and every automorphism $H \in \operatorname{Aut}_{F}\left(S_{f}\right)$ extends an inner automorphism of $D$. 
The fact that $D$ is a division algebra does not imply that $f$ is irreducible, so $S_{f}$ might not be a right division algebra.

Corollary 10. If the differential polynomial $f$ in Corollary 9 is irreducible, then $S_{f}$ is an infinite-dimensional right division algebra over $F$ and therefore does not have zero divisors.

If $f$ is an irreducible $A$-polynomial, it is not bounded by Theorem 2 .

Example 11. Suppose $F=\mathbb{R}$. The only central division algebra over $\mathbb{R}$ is $D=(-1,-1)_{\mathbb{R}}$. The function field $K$ of the projective real conic given by $x^{2}+y^{2}+z^{2}=0$ is a field extension of $\mathbb{R}$ in which $\mathbb{R}$ is algebraically closed and that splits $D$. There exists a derivation $\delta$ on $K$ with $\mathbb{R}=\operatorname{Const}(\delta)$. Thus there is an $A$-polynomial $f \in K[t ; \delta]$ of degree 2 , such that

$$
S_{f}=K[t ; \delta] / K[t ; \delta] f=K \oplus K t
$$

is an infinite-dimensional unital algebra over $\mathbb{R}$ with right nucleus $(-1,-1)_{\mathbb{R}}$, and left and middle nucleus $K$.

For $B=\operatorname{Mat}_{m}(\mathbb{R})$ and any field extension $K^{\prime}$ of $\mathbb{R}$ in which $\mathbb{R}$ is algebraically closed, with a derivation $\delta$ on $K^{\prime}$ such that $\mathbb{R}=\operatorname{Const}(\delta)$, there is a reducible $A$-polynomial $f \in K^{\prime}[t ; \delta]$ of degree $m$, such that

$$
S_{f}=K^{\prime}[t ; \delta] / K^{\prime}[t ; \delta] f
$$

is an infinite-dimensional unital algebra over $\mathbb{R}$ with right nucleus $B$ and left and middle nucleus $K^{\prime}$.

\section{Algebras whose right nucleus is a $p$-Algebra}

Let now $K$ be a field of characteristic $p>0$ together with a derivation $\delta$ on $K$. Put $R=K[t ; \delta]$ and $F=$ Const $(\delta)$. There are two cases which can occur: either $\delta$ is an algebraic derivation, or $\delta$ is transcendental which means $[K: F]=\infty$. We assume that $\delta$ is an algebraic derivation of degree $p^{e}$ with minimum polynomial

$$
g(t)=t^{p^{e}}+c_{1} t^{p^{e-1}}+\cdots+c_{e} t \in F[t]
$$

of degree $p^{e}$. Then $K=F\left(u_{1}, \ldots, u_{e}\right)=F\left(u_{1}\right) \otimes_{F} \cdots \otimes_{F} F\left(u_{e}\right)$ with $u_{i}^{p}=a_{i} \in F$ for all $i \in\{1, \ldots, e\}$, and $[K: F]=p^{e}$, that is $K$ is a finite purely inseparable field extension of exponent one and $K^{p} \subset F \subset K$. The center $C(R)$ of $R$ is $F[z]$ with $z=g(t)-d_{0}, d_{0} \in F$, and the two-sided elements in $R$ have the form $u h(t)$ with $u \in K^{\times}, h(t) \in C(R)$.

Recall that a central simple algebra $B=\operatorname{Mat}_{r}(D)$ over a field $F$ of characteristic $p$ is a $p$-algebra if it has index $p^{n}$, equivalently, if its exponent is a power of $p$ [11, p. 154].

Note that for $f(t)=g(t)-d \in F[t]$ (so $f(t)$ is two-sided in this case),

$$
(K, \delta, d)=K[t ; \delta] / K[t ; \delta] f(t)
$$

is an associative central simple $F$-algebra called a differential extension of $K$ and treated in $[11$, p. 23]. $K$ is a maximal subfield of $(K, \delta, d)$.

Theorem 12. [2, Lemma 20'] Let $B$ be a p-algebra of degree $m$ over $F$ which is split by a purely inseparable extension $K$ of exponent one (i.e., has exponent $p$ ), such that $m \leq[K: F]$. Then

$$
B \cong \operatorname{Nuc}_{r}\left(S_{f}\right)
$$


for some $f \in K[t ; \delta]$ of degree $m$ and a suitable $\delta$ with $F=\operatorname{Const}(\delta)$.

We start by looking at the case that $m=[K: F]=p^{e}$ and immediately obtain (i) and (ii) in the following result on $p$-algebras by employing only Theorem 1 (v) from Petit [14] and Amitsur's Theorem 12 (only the fact that then $B$ is cyclic uses Hood's Main Theorem [9, Main Theorem]):

Theorem 13. Let $B$ be a p-algebra of degree $m$ over $F$ which is split by a purely inseparable field extension $K$ of exponent one with $m=[K: F]$.

(i) There is an algebraic derivation $\delta$ on $K$ of degree $m$ with minimum polynomial $g(t)$ such that the center of $K[t ; \delta]$ is $F[z]$ with $z=g(t)-d_{0}, d_{0} \in F$, and

$$
B=\left(K, \delta, d_{0}\right)
$$

with $f(t)=g(t)-d_{0}$. B is a cyclic algebra.

(ii) $F[t] /(f)$ is a subfield of $B$ of degree $p^{e}$ over $F$ if and only if $f$ is irreducible in $F[t]$.

(iii) $f \in K[t ; \delta]$ is irreducible if and only if $B$ is a division algebra.

(iv) $B \cong \operatorname{Mat}_{p^{e}}(F)$ if and only if there is $b \in K$ such that

$$
d_{0}=V_{g}(b)=V_{p^{e}}(b)+c_{1} V_{p^{e-1}}(b)+\cdots+c_{e} b .
$$

Proof. (i) If $m=[K: F]$ then there is a differential polynomial $f \in K[t ; \delta]$ of degree $m$ and a suitable $\delta$ such that $B \cong \operatorname{Nuc}_{r}\left(S_{f}\right)$ by Theorem 12. Here $B$ is an associative subalgebra of $S_{f}$ of dimension $m^{2}$ and $S_{f}$ has dimension $m^{2}$ as well. Therefore $S_{f}=B$ is associative and $f \in K[t ; \delta]$ must be a two-sided differential polynomial of degree $m$, i.e. $B=K[t ; \delta] /(f)$ is a quotient algebra (Theorem $1(\mathrm{v})$ ). Without loss of generality we may assume $f$ is monic. Thus $f \in C(R)$ and since $f$ has degree $m=p^{e}$, we obtain that $f(t)=g(t)-d_{0}$ and so $B=\left(K, \delta, d_{0}\right) . K$ is a purely inseparable field extension of $F$ which is an (even maximal) subfield of $B$ splitting $B$, therefore $B$ is cyclic [9, Main Theorem].

(ii) Since here $f(t) \in F[t]$, we know that $F[t] /(f)$ is a subfield of $B$ of degree $p^{e}$ over $F$ if and only if $f$ is irreducible in $F[t]$.

(iii) is [8, Proposition 4] and (iv) is a consequence from (i) together with Theorem [11, Theorem 1.3.27].

Remark 14. Let us briefly put the previous result into context:

(i) Let A be a central simple $p$-algebra of degree $p^{n}$ over $F$. It is a well known classical result that $A$ is cyclic over $F$ if and only if $A$ has a subfield $K$ such that $K$ is a purely inseparable extension of $F$ and $K$ is a splitting field for A (this is [9, Main Theorem], which removed Albert's restriction that $K$ be simple from [1, Theorem (7.27)]).

(ii) Mammone characterized the central simple algebras split by a purely inseparable field extension $K$ of exponent one in [12]: in particular, if $B$ is a central simple algebra over $F$ of degree $m=p^{e}$ containing $K$ where $[K: F]=m$, then $B$ is a differential crossed product, that means $B$ contains a $K$-basis of the form $\left\{z_{1}^{i_{1}} \cdots z_{n}^{i_{n}} \mid 0 \leq i_{k} \leq p-1\right\}$ satisfying a kind of commutativity law with elements of $K$ which involves a set of $n F$-derivations of $K$. The algebra $B$ then yields elements $b_{i}=z_{i}^{p}$ and $u_{i j}=z_{i} z_{j}-z_{j} z_{i}$ in $K$. Conversely, given sets $B=\left\{b_{i} \mid i=1, \ldots, n\right\}$ and $U=\left\{u_{i j}: i, j=1, \ldots, n\right\}$ satisfying certain relations involving $F$-derivations of $K$, then $(U, B)$ arises from such a differential crossed product. 
In case $m<[K: F]=p^{e}$ we obtain a nonassociative algebra of dimension $m p^{e}$ containing $B$ as right nucleus:

Theorem 15. Let $B$ be a p-algebra of degree $m$ over $F$ which is split by a purely inseparable extension $K$ of exponent one such that $m<[K: F]$.

(i) There is an algebraic derivation $\delta$ and a differential polynomial $f \in K[t ; \delta]$ of degree $m$ such that

$$
S_{f}=K[t ; \delta] / K[t ; \delta] f
$$

is an algebra over $F$ of dimension $m p^{e}$ with right nucleus $B$, left and middle nucleus $K$, and nucleus $\operatorname{Nuc}\left(S_{f}\right)=B \cap K$ an intermediate field of $K / F$, unequal to $K$.

(ii) $f$ is irreducible if and only if $B$ is a division algebra, if and only if $S_{f}$ is a division algebra.

(iii) Every automorphism $H \in \operatorname{Aut}_{F}\left(S_{f}\right)$ extends an inner automorphism of $B$ and an automorphism of $K$.

Proof. (i) The existence of a suitable $f$ follows from Theorem 12 and the statements on the left and middle nuclei from Theorem 1 . Since $f$ is not two-sided, $K$ is not contained in the right nucleus of $S_{f}$, i.e. not contained in $B$ [15, Theorem 9]. Thus $\operatorname{Nuc}\left(S_{f}\right)=B \cap K$ is properly contained in $K$, so that it is an intermediate field of the field extension $K / F$.

(ii) By Proposition 3 and Theorem $1, f$ is irreducible if and only if $B$ is a division algebra, if and only if $S_{f}$ is a division algebra.

(iii) An automorphism of $S_{f}$ extends both an inner automorphism of $B$ and an automorphism of $K$ by Remark 6 .

Corollary 16. Let $D$ be a division p-algebra of degree $m$ over $F$ which is split by a purely inseparable extension $K$ of exponent one such that $m<[K: F]$. Then there is an irreducible polynomial $f \in K[t ; \delta]$ of degree $m$ such that $S_{f}$ is a division algebra over $F$ of dimension $m p^{e}$ with right nucleus $D$, left and middle nucleus $K$, and nucleus $D \cap K$ an intermediate field of $K / F$, unequal to $K$.

The fact that $f$ is irreducible in Corollary 16 follows from Proposition 3. Note that every division $p$-algebra over $F$ split by $K$ has degree $m \leq[K: F]$, so that Theorem 13 (iii) and Corollary 16 cover all possible cases for a division $p$-algebra.

We could ask for the algebra $S_{f}$ of smallest possible dimension which contains a given central simple algebra $B$ as a right nucleus. This is equivalent to asking for a purely inseparable extension $K$ of exponent one splitting $B$ of smallest possible degree $[K: F]=p^{e}$ satisfying $m<[K: F]$, which in turn is connected to the question how many cyclic algebras are needed when saying that $B$ is similar to a product of cyclic algebras of degree $p$ in the Brauer group $B r(F)$.

Theorem 17. Let $B$ be a p-algebra over $F$ of degree $m$, index $d=p^{n}$ and exponent $p$, such that $m=r^{2} p^{n}<p^{d-1}$. Then there is a purely inseparable extension $K$ of exponent one with $[K: F]=p^{d-1}$, and a differential polynomial $f \in K[t ; \delta]$ of degree $m$ such that

$$
S_{f}=K[t ; \delta] / K[t ; \delta] f
$$


is an algebra over $F$ of dimension $m p^{d-1}$ with right nucleus $B$ and the properties listed in Theorem 15.

Proof. Let $B$ be a $p$-algebra of index $p^{n}$ and exponent $p$. Then there is a purely inseparable field extension $K / F$ of exponent one with $K=F\left(u_{1}, \ldots, u_{d-1}\right), u_{i}^{p}=a_{i} \in F$, and $[K: F]=$ $p^{d-1}$, which splits $B$ [7, Theorem 1.1.]. We have $m=r^{2} d=r^{2} p^{n}$ for some $r \geq 1$.

We need $m=r^{2} p^{n} \leq[K: F]=p^{d-1}$ to be able to apply Theorem 12. By Theorem 12 this implies that $B \cong \operatorname{Nuc}_{r}\left(S_{f}\right)$ for some $f \in K[t ; \delta]$ of degree $m$ and a suitable $\delta$ with $F=\operatorname{Const}(\delta)$. Since each $f \in K[t ; \delta]$ is bounded by Proposition $3, B=\operatorname{Nuc}_{r}\left(S_{f}\right)$ is a division algebra if and only if $f$ is irreducible [8, Proposition 4], if and only if $S_{f}$ is a division algebra.

We obtain that for a division algebra $D$, the smallest possible dimension $l$ of a division algebra $S_{f}$ containing $D$ as right nucleus satisfies $m^{2}<l=m p^{e} \leq m p^{m-1}$ :

Corollary 18. Let $D$ be a division p-algebra of degree $m$ and exponent $p$ over $F$. Then there is a purely inseparable extension $K$ of exponent one with $[K: F]=p^{m-1}$, and an irreducible differential polynomial $f \in K[t ; \delta]$ of degree $m$ such that

$$
S_{f}=K[t ; \delta] / K[t ; \delta] f
$$

is a division algebra over $F$ of dimension $m p^{m-1}$ with right nucleus $D$ and the properties listed in Theorem 15.

Proof. There is a purely inseparable field extension $K=F\left(u_{1}, \ldots, u_{m-1}\right)$ of exponent one, $u_{i}^{p}=a_{i} \in F$, and $[K: F]=p^{m-1}$, which splits $D$ [7, Theorem 1.1.].

We need $m=p^{n} \leq[K: F]=p^{m-1}$ to be able to apply Theorem 12. This holds for all prime $p$ and $n \geq 1$ as it is equivalent to $n \leq p^{n}-1$, i.e. to $n+1 \leq p^{n}$, which is true for all prime $p$ and $n \geq 1$. Therefore there is a purely inseparable field extension $K / F$ of exponent one with $m \leq[K: F]=p^{m-1}$ which splits $D$. By Theorem 12 this implies that $B \cong \operatorname{Nuc}_{r}\left(S_{f}\right)$ for some $f \in K[t ; \delta]$ of degree $m$ and a suitable $\delta$ with $F=\operatorname{Const}(\delta)$. Since $D$ is a division algebra and $f$ bounded, $f$ is irreducible and $S_{f}$ is a division algebra.

Acknowledgement: We would like to thank the referee for their kind and thorough report which greatly helped to improve the paper.

\section{REFERENCES}

[1] A. A. Albert, "Structure of algebras." Revised printing, Amer. Math. Soc., Providence, R.I., 1961.

[2] A. S. Amitsur, Differential polynomials and division algebras. Annals of Mathematics, Vol. 59 (2) (1954) 245-278.

[3] A. S. Amitsur, Generic splitting fields of central simple algebras. Ann. of Math. 62 (2) (1955), 8-43.

[4] M. Boulagouaz, A. Leroy, $(\sigma, \delta)$-codes. Adv. Math. Commun. 7 (4) (2013), 463-474.

[5] J. Carcanague, Idéaux bilatères d'un anneau de polynômes non commutatifs sur un corps. J. Algebra (18) $1971,1-18$.

[6] J. Carcanague, Quelques résultats sur les anneaux de Ore. C. R. Acad. Sci. Paris Sr. A-B 269 (1969), A749-A752.

[7] M. Florence, On the symbol length of p-algebras. Compositio Mathematica 149 (8) (2013), 1353-1363. 
[8] J. Gòmez-Torrecillas, Basic module theory over non-commutative rings with computational aspects of operator algebras. With an appendix by V. Levandovskyy. Lecture Notes in Comput. Sci. 8372, Algebraic and algorithmic aspects of differential and integral operators, Springer, Heidelberg (2014) 23-82.

[9] J. M. Hood, Central simple p-algebras with purely inseparable subfields. J. Algebra 17 (1971) 299-301.

[10] N. Jacobson, The Theory of Rings, AMS, Providence, RI, 1943

[11] N. Jacobson, "Finite-dimensional division algebras over fields." Springer Verlag, Berlin-Heidelberg-New York, 1996.

[12] P. Mammone, Remarques sur les produits croisés différentiels. Acad. Roy. Belg. Bull. Cl. Sci. (5) 68 (1982), no. 10, 651-664.

[13] A. Nowicki, Rings and fields of constants for derivations in characteristic zero. J. Pure Appl. Algebra 96 (1) (1994), 47-55.

[14] J.-C. Petit, Sur certains quasi-corps généralisant un type d'anneau-quotient. Séminaire Dubriel. Algèbre et théorie des nombres 20 (1966 - 67), 1-18.

[15] S. Pumplün, Nonassociative differential extensions of characteristic $p$. Results in Mathematics 72 (1-2) (2017), 245-262. DOI 10.1007/s00025-017-0656-x

[16] J.-C. Petit, Sur les quasi-corps distributifs à base monogène. C. R. Acad. Sc. Paris 266 (1968), Série A, 402-404.

[17] R. D. Schafer, "An Introduction to Nonassociative Algebras.” Dover Publ., Inc., New York, 1995.

E-mail address: susanne.pumpluen@nottingham.ac.uk

School of Mathematical Sciences, University of Nottingham, University Park, Nottingham NG7 2RD, United KingDOM 